\title{
Usabilidad de tablets para el acceso a campus virtuales universitarios por alumnos con discapacidad
}

\author{
Ana Millán JimÉneZ \\ Universidad de Murcia \\ amillan@um.es \\ Antonio PÉREz MANZANO \\ Universidad de Murcia \\ aperez@um.es
}

\begin{abstract}
Resumen:
Es habitual que los alumnos con discapacidad, por sus propias características, tengan problemas para acceder a los campus virtuales universitarios, donde se encuentran los contenidos y herramientas académicas necesarias para superar las asignaturas y obtener su graduación.

En este arículo presentamos los resultados de una investigación sobre la usabilidad de distintos tipos de tablets por parte de este tipo de alumnado, con el objetivo de evaluar la adecuación más correcta de aquellas, en la acceso y tratamiento de tales recursos virtuales.
\end{abstract}

Palabras clave: Usabilidad; tablets; discapacidad; campus virtual.

\section{Usability of tablets for accessing virtual university campuses by students with disabilities.}

\begin{abstract}
:
Usually, by their own characteristics, disabled students have trouble using virtual university campuses. And contents and necessary tools to pass subjects and to obtain graduation are usually stored there. We present here the results of an investigation into the usability of different types of tablets by these students. Our aim is to evaluate the adequacy of those devices in the access and processing of such virtual resources.
\end{abstract}

Key Words: Usability; tablets; disability; virtual campuses.

\section{Referencia normalizada:}

Millán Jiménez, A. y Pérez Manzano, A. (2014): Usabilidad de tablets para el acceso a campus virtuales universitarios por alumnos con discapacidad. Historia y Comunicación Social. Vol. 19. Núm. Especial Febrero. Págs. 805-818.

Sumario: 1. Introducción, 2. Objetivos de la investigación, 3. Metodología utilizada, 4. Análisis e interpretación de los resultados, 4.1 Valoración de cada ítem evaluado para cada tipo de tablets, 4.2 Valoración global de la usabilidad de las tablets, 4.3 Valoración de la usabilidad de cada tablet en función de la discapacidad del alumnado, 4.4. Evaluación del entorno virtual de la Universidad de Murcia, 5. Bibliografía 


\section{Introducción}

Vivimos en una sociedad definida por espacios virtuales y tecnológicos que modifican nuestra percepción y relación con el entorno, conviertiendo en accesibles dimensiones con las que interactuamos de manera inmediata y a una velocidad hasta ahora inimaginable.

Esa misma rapidez con la que esas tecnologías nos permiten actuar, es con la que aparecen y desaparecen de los mercados, obligando a permanecer en un constante estado de alerta y en una contínuo recliclaje de conocimientos, si no queremos quedarnos al margen (Bernete, 2010). Por eso es necesario actualizar nuestro saber sobre las nuevas tecnologías y su repercusión en la población, en este caso concreto, la población universitaria con discapacidad. La relación entre sociedad y nuevas tecnologías es siempre apasionante.

El uso de las TIC por los diferentes grupos sociales depende de las infraestructuras, las capacidades, y los diferentes factores que configuran nuestro entorno social e individual, y que dinamizan o enlentecen la apropiación de las tecnologías más innovadoras (Bernete, 2010). Un amplio segmento de los jóvenes españoles utilizan la red para diferentes actividades de su vida cotidiana. Y, como no podía ser de otro modo, el colectivo universitario también se encuentra en esa franja de población en la que el acceso y manejo de las nuevas tecnologías es habitual (Víctor Pérez- Díaz y Juan Carlos Rodríguez, 2008). Es inegable el papel tan decisivo que tienen las redes en la organización social de la juventud. Suponen una auténtica revolución en las sociedades avanzadas, especialmente en el ámbito de la comunicación y de las relaciones sociales (Pérez, 2010:70).

La información y los datos que nos muestra Cientouno (empresa de Estrategia y Marketing en Redes Sociales) son apabullantes y confirman que en España, Facebook cuenta con más de ocho millones de usuarios, y que de las cuatro palabras más buscadas en Internet, tres son el nombre de redes sociales: Facebook, Tuenti y Twitter. Este mismo estudio afirma que el $85 \%$ de los internautas pertenece a alguna red social, y que el $65 \%$ las consulta todos los días. Asimismo, se concluye que el $10 \%$ del tiempo pasado en Internet se pasa en redes sociales y que los usuarios de Tuenti le dedican 80 minutos al día. Sólo en España, Tuenti tiene un $35 \%$ más de tráfico que Google (Sotelo, 2011:117).

Por otra parte, el último informe presentado por la Fundación Telefónica sobre "La Sociedad de la Información en España 2009", expone que el uso de las redes sociales entre los jóvenes se ha multiplicado por seis a lo largo de 2009, lo que supone un aumento del 500\% con respecto al mismo período del año anterior (Bernete, 2010).

Después de constatar todos estos datos resulta muy fácil deducir que estas conductas digitales no pueden dejar de afectar al modo

en el que los jóvenes gestionan su vida. En estos espacios pueden mostrarse como quieran sin preocuparse por la reacción del otro. Sin embargo, el uso tan continuado de estas vías para relacionarse con los demás perjudica el desarrollo de habilidades 
sociales. La práxis casi exclusiva de este tipo de comunicación puede desembocar en una especie de incapacidad relacional que les dificultará el camino cuando deban interactuar física y personalmente con los demás (Ferrán Ramón-Cortés, 2010). Las mismas relaciones familiares se ven afectadas por el abuso de estos medios, y no nos referimos sólo a la utilización de las nuevas tecnologías, sino también a los más clásicos como son el teléfono, la radio o la televisión (Lorente, Bernete, y Becerril, 2004).

Las instituciones educativas no podían quedarse al margen de esta invasión tecnológica. En todos los niveles educativos es muchísimo más frecuente la accesibilidad y el manejo de las TICs por parte del alumnado. Además, seríamos muy ingenuos si pensáramos que únicamente se utilizan. En absoluto, son mucho más. Son portadoras de nuevos vocablos, conceptos, valores y pautas de comportamiento. A la vez que presionan para transformar los modos de enseñar y aprender, insistiendo en la incorporación en las aulas estas tecnologías (Bernete, 2010).

Desde estos presupuestos, hemos prestado una especial atención al uso de las nuevas tecnologías en el ámbito educativo universitario, realizando una investigación en la que se estudia y evalua el uso que sus alumnos (con algún tipo de discapacidad visual, auditiva, física o psíquica) hacen de las nuevas computadoras portátiles: las tablets. Concretamente de cuatro modelos diferentes de tablets: iPad, Toshiba, Samsung Galaxy Tab y Samsung Galaxy Note. En este artículo se analizan los resultados obtenidos.

\section{Objetivos de la investigación}

Con el fin de organizar las actuaciones a desarrollar en la investigación y en base a las necesidades detectadas con anterioridad, planteamos los siguientes objetivos:

- Analizar la usabilidad percibida de las tablets como medio de acceso y utilización de un entorno virtual universitario por alumnos con diferentes tipos de discapacidad.

- Identificar qué modelo de tablet, de los de mayor éxito en el mercado, se adapta mejor al alumnado en función de su discapacidad.

- Evaluar el desempeño de este colectivo en el acceso y búsqueda de contenidos académicos habituales en la página web de la Universidad de Murcia

\section{Metodología utilizada}

Hemos utilizado una metodología mayoritariamente cuantitativa, combinada con información cualitativa obtenida con los participantes durante el estudio. 
Fases del proceso:

Fase inicial: De la base de datos de alumnos con discapacidad de la Universidad de Murcia, se seleccionan al azar un listado de alumnos con diferentes tipos de discapacidades organizadas en cuatro bloques: discapacidad visual, auditiva, física con problemas de manipulación y, por último, necesidades educativas especiales (dislexias y similar). Posteriormente se confirma telefónicamente el interés por participar en la investigación.

Fase 2: Entrega consecutiva de los cuatro modelos de tablets a los participantes con la consiguiente cumplimentación del cuestionario vía online, a través del portal www.encuestas.um.es

Fase 3: Introducción de los datos obtenidos en los cuestionarios al programa estadístico SPSS y análisis de los mismos.

Fase 4: Obtención de resultados y elaboración de conclusiones.

Muestra del estudio: Alumnos matriculados en la Universidad de Murcia en el curso 2012/2013 que acuden a la unidad de Atención a la Discapacidad y Voluntariado $(\mathrm{ADyV})$ por presentar algún tipo de discapacidad.

El estudio se ha realizado con once alumnos/as, tres de ellos con discapacidad auditiva, dos con resto visual, dos con discapacidad física, uno con dislexia, uno con trastorno bipolar, uno con Síndrome de Asperger y otro con manipulación reducida.

La técnica empleada para la recogida de datos empíricos ha sido el cuestionario, compuesto por:

Dieciséis actividades relacionadas con tareas que habitualmente se realizan a través del entorno virtual de la Universidad de Murcia. Estas actividades incorporaban la ruta de acceso a diferentes servicios, que los participantes debían seguir y evaluarla mediante una escala de valoración tipo Licker con cinco opciones de respuesta: $1=$ Totalmente en desacuerdo, $2=$ En desacuerdo, $3=$ Indiferente, $4=$ De acuerdo, $5=$ Totalmente de acuerdo.

Dos preguntas de evaluación:

- La primera dirigida a evaluar una serie de ítems relacionados con la usabilidad de las tablets, en una escala de gratificación del 1 al 10 (siendo el valor 1 el más bajo y el 10 el más alto). Los ítems a evaluar han sido: el manejo del teclado táctil, la dimensión de la pantalla, el acceso al menú principal, la capacidad de almacenamiento, la velocidad de navegación, el acceso al menú principal, la estética del tablet, el contraste e iluminación, la comodidad y la duración de la batería.

- La segunda destinada a valorar la utilidad de algunos de los servicios que incorpora la página web de la Universidad de Murcia y que han sido trabajados en las 16 actividades anteriores. Para ello, se ha aplicado una escala de valora- 
ción tipo Licker con cuatro opciones de respuesta donde 1= Ninguna utilidad, 2= Poca utilidad, $3=$ Bastante utilidad y $4=$ Mucha utilidad.

Tres preguntas abiertas que permitían al encuestado, por un lado contestar cualquier otra cosa, y por otro lado proporcionaba a nuestro estudio una mayor riqueza de detalle en las contestaciones

\section{Análisis e interpretación de los resultados}

Usabilidad de las tablets en el entorno virtual de la Universidad de Murcia en función de la discapacidad del alumnado.

4.1. Valoración de cada ítem evaluado para cada tipo de tablets.

\begin{tabular}{|c|c|c|c|c|c|c|c|c|}
\hline & \multicolumn{8}{|c|}{ TABLET } \\
\hline & \multicolumn{2}{|c|}{ iPAD } & \multicolumn{2}{|c|}{ Toshiba } & \multicolumn{2}{|c|}{ Samsung Galaxy } & \multicolumn{2}{|c|}{ Samsung Note } \\
\hline & Media & Mediana & Media & Mediana & Media & Mediana & Media & Mediana \\
\hline Teclado & 9,80 & 10 & 7,80 & 8 & 7,70 & 9 & 8,20 & 9 \\
\hline Pantalla & 9,30 & 10 & 8,20 & 8 & 9,00 & 10 & 8,40 & 8 \\
\hline Menú principal & 8,60 & 9 & 7,60 & 8 & 7,30 & 7 & 7,80 & 8 \\
\hline Almacenamiento & 7,70 & 8 & 8,40 & 9 & 7,50 & 7 & 8,40 & 8 \\
\hline Velocidad & 9,20 & 10 & 5,70 & 5 & 6,90 & 7 & 7,10 & 7 \\
\hline $\begin{array}{c}\text { Menú principal } \\
\text { tablet }\end{array}$ & 8,50 & 9 & 7,70 & 8 & 7,10 & 7 & 7,50 & 8 \\
\hline Estética & 9,30 & 9 & 7,90 & 9 & 8,80 & 9 & 8,00 & 8 \\
\hline Comodidad & 9,40 & 10 & 7,60 & 7 & 8,80 & 10 & 8,50 & 9 \\
\hline $\begin{array}{l}\text { Contraste e } \\
\text { Iluminación }\end{array}$ & 9,80 & 10 & 8,30 & 9 & 9,40 & 10 & 8,80 & 9 \\
\hline Batería & 8,60 & 9 & 7,60 & 8 & 7,40 & 8 & 7,80 & 8 \\
\hline
\end{tabular}

Tabla 1. Valoración de ítems por tipo de tablets 
Gráfico 1. Valoración de ítems por tipo de tablets

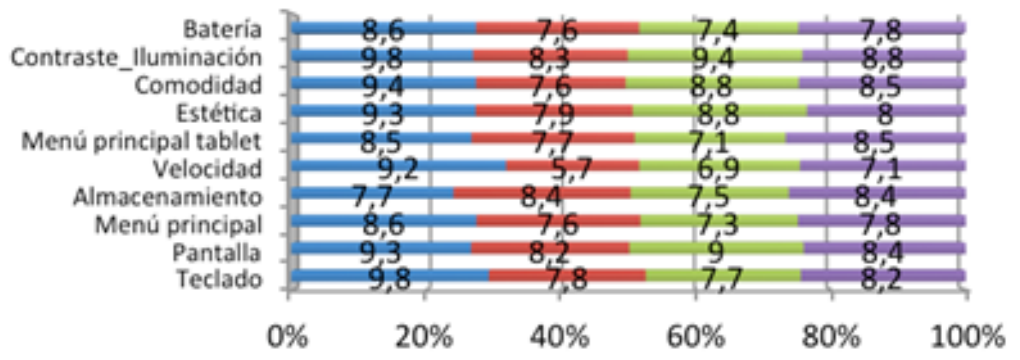

IPAD $=$ TOSHIBA $=$ SAMSUNG GALAXY

En relación a la valoración concreta de cada tipo de tablets evaluada podemos concluir lo siguiente.

El Apple iPad ha sido el modelo que muestra una mayor puntuación global, además es señalado como el modelo que aporta mayor facilidad de conexión a la red. No obstante, queda rezagado en lo que se refiere al almacenamiento, quedando por encima otras tablets como por ejemplo el Samsung Galaxy Note.

En relación a las carencias detectadas por la muestra en los equipos evaluados, señalan, por ejemplo que para la mayoría, las baterías tienen una duración excasa, siendo el iPad la tablet que menos problemas da en este aspecto. También se detecta una opinión generalizada que apunta al modelo Toshiba como el que tiene una velocidad de navegación más lenta, con una puntuación de 5,7 sobre 10, estando por encima de éste el resto de tablets con una puntuación superior al 7. Esta variable marcará diferencias negativas para el modelo de Toshiba en tareas posteriores. La lentitud de acceso a la web es algo señalado también tras el uso del Samsung Galaxy Note.

4.2. Valoración global de la usabilidad de las tablets.

\begin{tabular}{|c|c|}
\hline & $\begin{array}{c}\text { Total } \\
\text { usabilidad }\end{array}$ \\
\cline { 2 - 3 } Media & \\
\hline iPad & 9,02 \\
\hline Toshiba & 7,68 \\
\hline Samsung Galaxy & 7,99 \\
Samsung Note & 8,05 \\
\hline
\end{tabular}

Tabla 2. Usabilidad global por tipo de tablets 
Gráfico 2. Usabilidad global por tipo de tablets

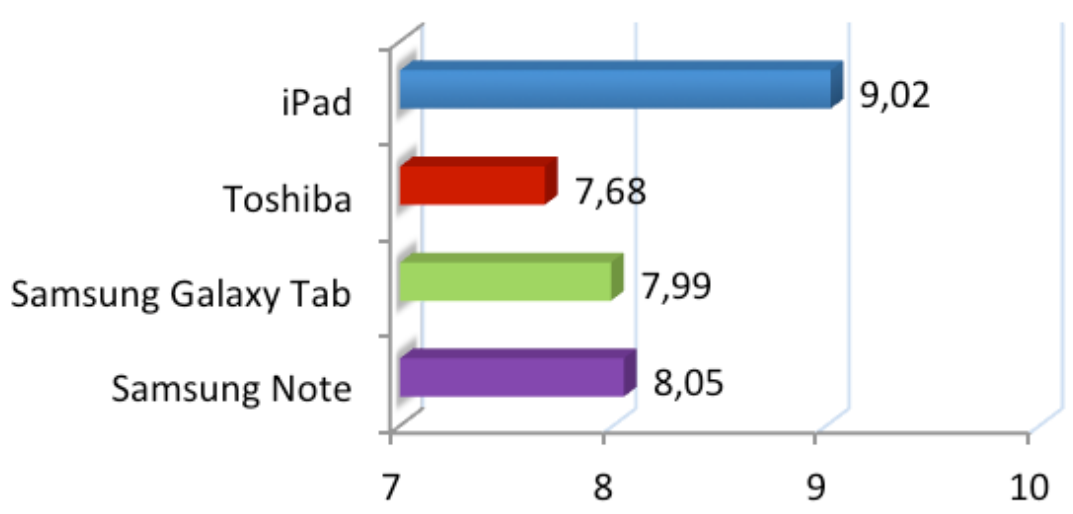

Una carencia generalizada en todos los modelos de tablets ha sido la ausencia de un puerto USB en todos los modelos, impidiendo, por consiguiente, el uso de pendrives, lo que supondría, en temas de comparación con otros materiales como el portátil, un aspecto en contra. Ninguno de los participantes señala conocer la posibilidad de conectar prendrives a las tablets mediante adaptadores USB al efecto.

A nivel global, observamos que la tablet que mejor aprobación ha tenido entre los alumnos con discapacidad ha sido el modelo de Apple iPad, obteniendo una puntuación de 9,02 sobre 10. Por el contrario, el modelo que menos entusiasmo ha generado ha sido el modelo Toshiba con un 7,68 (severamente castigado por su lentitud en las tareas en red).

La muestra ha manifestado diferentes elementos que benefician a las tablets en comparación con los, también habituales en las aulas, netbooks:

- Mejor resolución en documentos y fotografías que impresas en papel.

- Más ligeras y mayor contraste y nitidez.

- Un tamaño perfecto para ser manejadas y transportadas.

- Buena organización, sencilla y cómoda, de los iconos y contenidos en pantalla.

- Facilita la navegación siempre que la conexión a la red sea la adecuada. 
4.3. Valoración de la usabilidad de cada tablet en función de la discapacidad del alumnado.

Tabla 3. Usabilidad por tipo de discapacidad

\begin{tabular}{|c|c|c|c|c|c|c|c|c|c|}
\hline & \multicolumn{2}{|c|}{ Discapacidad auditiva } & \multicolumn{2}{|c|}{ Discapacidad visual } & \multicolumn{2}{|c|}{ Discapacidad fisica } & \multicolumn{2}{|c|}{ NEE } \\
\hline & & Media & Mediana & Media & Mediana & Media & Mediana & Media & Mediana \\
\hline \multirow[t]{10}{*}{$\underline{\mathrm{PAD}}$} & USAB_teclado & 10,00 & 10 & 10,00 & 10 & 10,00 & 10 & 9,50 & 10 \\
\hline & USAB_pantalla & 9,33 & 10 & 10,00 & 10 & 9,00 & 9 & 9,25 & 10 \\
\hline & USAB_menú_principal & 9,00 & 9 & 5,00 & 5 & 8,50 & 9 & 9,25 & 10 \\
\hline & USAB_almacenamiento & 6,33 & 8 & 8,00 & 8 & 7,00 & 7 & 9,00 & 10 \\
\hline & USAB_velocidad & 9,33 & 10 & 10,00 & 10 & 9,50 & 10 & 8,75 & 10 \\
\hline & USAB_menú_principTablet & 8,00 & 9 & 5,00 & 5 & 9,50 & 10 & 9,25 & 10 \\
\hline & USAB_estética & 8,67 & 9 & 10,00 & 10 & 9,00 & 9 & 9,75 & 10 \\
\hline & USAB_comodidad & 9,67 & 10 & 8,00 & 8 & 9,00 & 9 & 9,75 & 10 \\
\hline & USAB_contrast_iluminacion & 10,00 & 10 & 10,00 & 10 & 9,50 & 10 & 9,75 & 10 \\
\hline & USAB_batería & 9,33 & 9 & 10,00 & 10 & 8,50 & 9 & 7,75 & 9 \\
\hline \multirow[t]{10}{*}{ Thosiba } & USAB_teclado & 7,33 & 8 & 8,00 & 8 & 5,00 & 5 & 8,60 & 10 \\
\hline & USAB_pantalla & 7,67 & 8 & 8,00 & 8 & 7,00 & 7 & 8,80 & 9 \\
\hline & USAB_menú_principal & 6,67 & 7 & 7,00 & 7 & 9,00 & 9 & 8,00 & 8 \\
\hline & USAB_almacenamiento & 7,67 & 7 & 9,00 & 9 & 9,00 & 9 & 8,60 & 9 \\
\hline & USAB_velocidad & 6,67 & 7 & 4,00 & 4 & 3,00 & 3 & 6,00 & 5 \\
\hline & USAB_menú_principTablet & 6,33 & 7 & 9,00 & 9 & 7,00 & 7 & 8,40 & 9 \\
\hline & USAB_estética & 6,67 & 7 & 9,00 & 9 & 7,00 & 7 & 8,60 & 9 \\
\hline & USAB_comodidad & 7,00 & 7 & 7,00 & 7 & 6,00 & 6 & 8,40 & 9 \\
\hline & USAB_contrast_iluminacion & 8,00 & 7 & 9,00 & 9 & 8,00 & 8 & 8,40 & 9 \\
\hline & USAB_batería & 8,33 & 9 & 9,00 & 9 & 7,00 & 7 & 7,00 & 7 \\
\hline \multirow[t]{10}{*}{ Samsung Galaxy } & USAB_teclado & 8,67 & 9 & 8,00 & 8 & 3,00 & 3 & 8,00 & 9 \\
\hline & USAB_pantalla & 9,00 & 9 & 10,00 & 10 & 6,00 & 6 & 9,40 & 10 \\
\hline & USAB_menú_principal & 7,33 & 7 & 9,00 & 9 & 6,00 & 6 & 7,20 & 7 \\
\hline & USAB_almacenamiento & 7,33 & 7 & 10,00 & 10 & 8,00 & 8 & 7,00 & 7 \\
\hline & USAB_velocidad & 8,33 & 8 & 7,00 & 7 & 4,00 & 4 & 6,60 & 7 \\
\hline & USAB_menú_principTablet & 8,00 & 8 & 10,00 & 10 & 7,00 & 7 & 6,00 & 6 \\
\hline & USAB_estética & 7,67 & 8 & 9,00 & 9 & 8,00 & 8 & 9,60 & 10 \\
\hline & USAB_comodidad & 9,00 & 10 & 10,00 & 10 & 4,00 & 4 & 9,40 & 9 \\
\hline & USAB_contrast_iluminacion & 9,33 & 10 & 10,00 & 10 & 7,00 & 7 & 9,80 & 10 \\
\hline & USAB_batería & 8,33 & 8 & 10,00 & 10 & 8,00 & 8 & 6,20 & 6 \\
\hline \multirow[t]{10}{*}{ Samsung Note } & USAB_teclado & 7,33 & 8 & 9,00 & 9 & 8,00 & 8 & 8,60 & 9 \\
\hline & USAB_pantalla & 7,67 & 7 & 8,00 & 8 & 8,00 & 8 & 9,00 & 9 \\
\hline & USAB_menú_principal & 7,00 & 7 & 7,00 & 7 & 9,00 & 9 & 8,20 & 9 \\
\hline & USAB_almacenamiento & 7,33 & 8 & 9,00 & 9 & 10,00 & 10 & 8,60 & 8 \\
\hline & USAB_velocidad & 7,67 & 8 & 5,00 & 5 & 7,00 & 7 & 7,20 & 7 \\
\hline & USAB_menú_principTablet & 7,00 & 7 & 7,00 & 7 & 8,00 & 8 & 7,80 & 8 \\
\hline & USAB_estética & 6,33 & 6 & 9,00 & 9 & 8,00 & 8 & 8,80 & 9 \\
\hline & USAB_comodidad & 7,00 & 6 & 9,00 & 9 & 10,00 & 10 & 9,00 & 9 \\
\hline & USAB_contrast_iluminacion & 7,33 & 6 & 9,00 & 9 & 10,00 & 10 & 9,40 & 9 \\
\hline & USAB_batería & 7,67 & 8 & 9,00 & 9 & 7,00 & 7 & 7,80 & 8 \\
\hline
\end{tabular}


Gráfico 3. Usabilidad en discapacidad auditiva

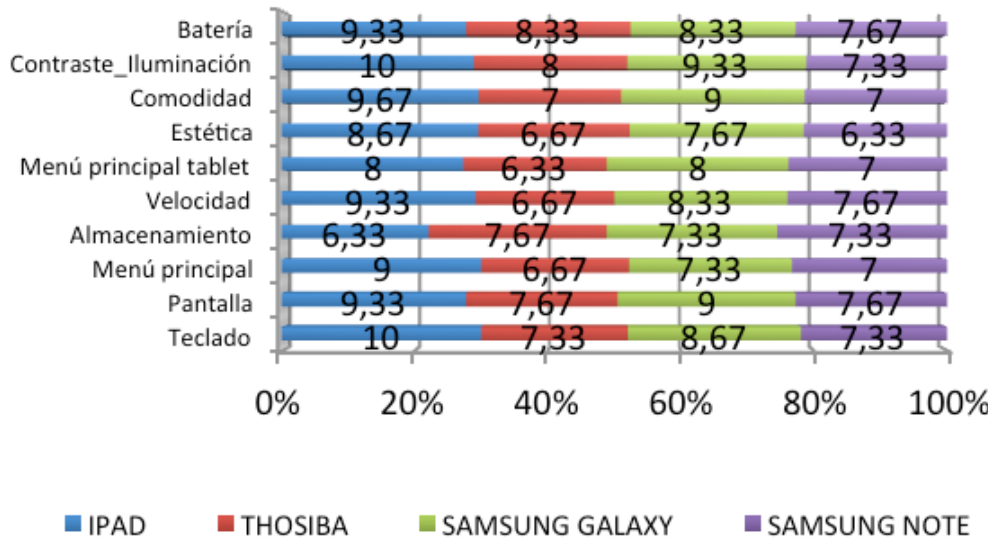

Gráfico 4. Usabilidad en discapacidad visual

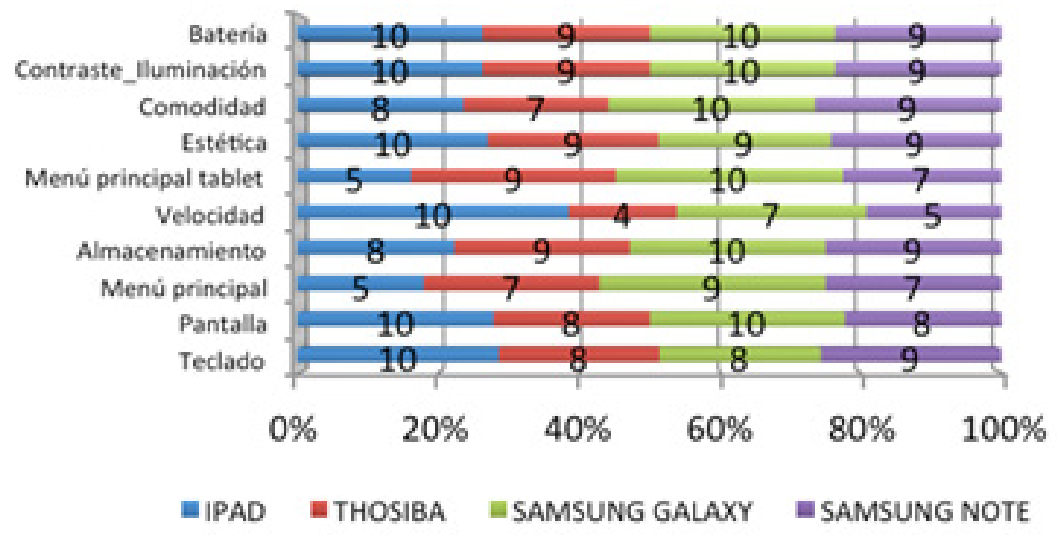


Gráfico 5. Usabilidad en discapacidad física

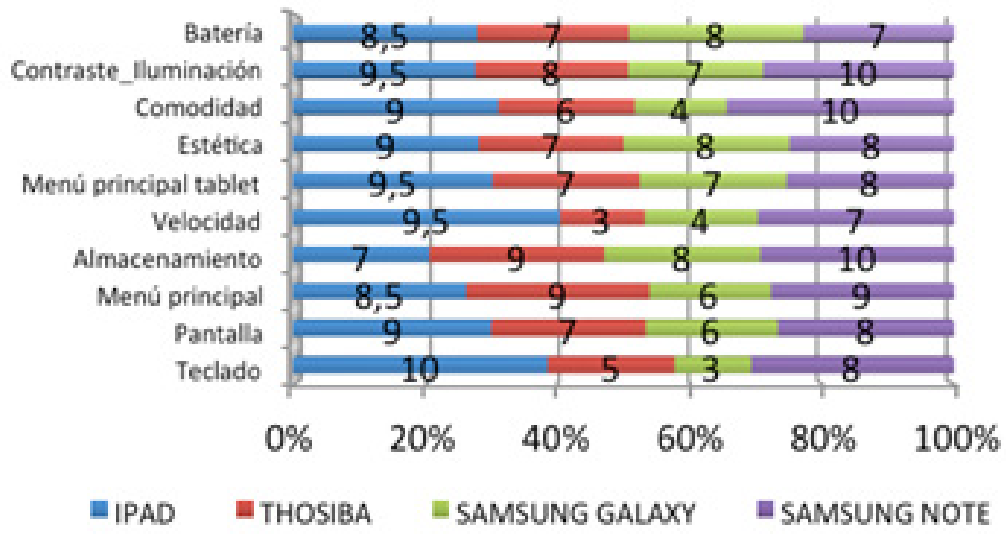

Gráfico 6. Usabilidad en necesidades educativas especiales

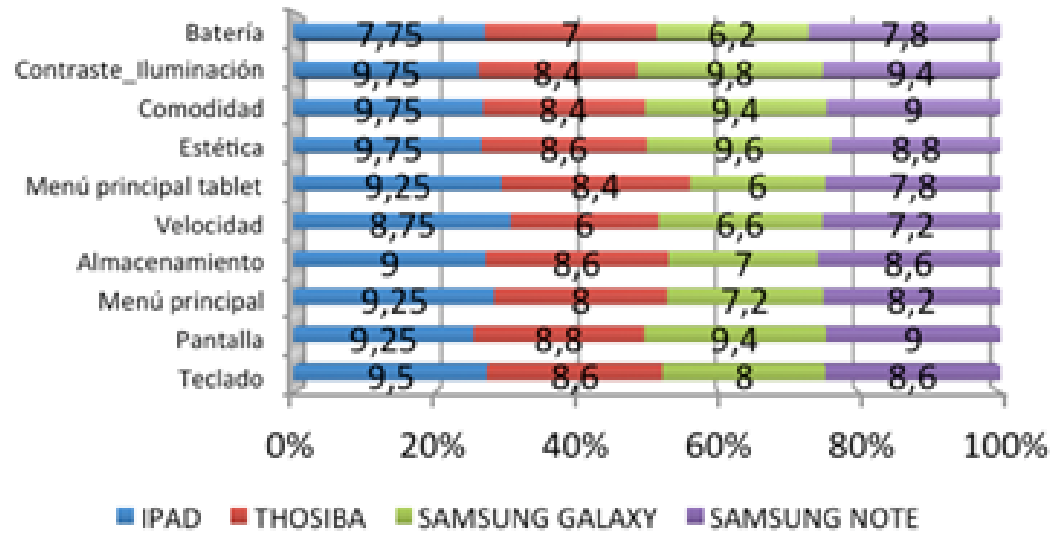

De la combinación entre las variables "tablets" y "tipo de discapacidad" con todos los ítems evaluados se han destacado los siguientes resultados.

En primer lugar, es interesante destacar que los alumnos con discapacidad visual han puntuado con una nota de 10 al Samsung Galaxy Tab en relación a la comodidad del tablet. Sin embargo, para este mismo ítem de evaluación, los alumnos con discapacidad física han puntuado a dicho tablet con un 4, dándole la mayor puntuación al Samsung Galaxy Note. Estos últimos también otorgan la menor puntuación en relación al teclado táctil y a la pantalla del Samsung Galaxy Tab. 
En otro orden de cosas, en relación al uso que estos alumnos dan a la página web de la UMU, se han obtenidos los siguientes resultados:

- Para las personas que presentan discapacidad visual, el "tamaño de las letras" de algunos enlaces de la página web de la Universidad de Murcia es demasiado pequeño, haciendo más difícil su manejo y acceso por estos alumnos. Por ejemplo el acceso a "Tutorías" y sus diferentes carpetas de mensajes recibidos o enviados.

- La distribución de los enlaces de la página web de la Universidad de Murcia dificulta el acceso a la información por parte de los alumnos. La mayoría de los diferentes listados de tareas y/o enlaces de acceso a los recursos de la web están muy apiñados. Por tanto, si accedemos a la web a través de dispositivos portátiles táctiles esta limitación se intensifica aun más, ya que disminuye la precisión a la hora de pulsar el enlace deseado.

- Títulos claramente señalados. En muchas ocasiones, la forma en la que la web de la UMU ofrece la información dificulta el acceso a la misma por parte de los estudiantes. Ejemplo de ello son la "Guía docente de una asignatura" o el "Horario", donde los títulos que dan acceso a esta información no quedan claros para el alumnado, especialmente para aquellos que tienen dificultad de comprensión lectura, como es el caso de los alumnos con discapacidad auditiva o necesidades educativas especiales.

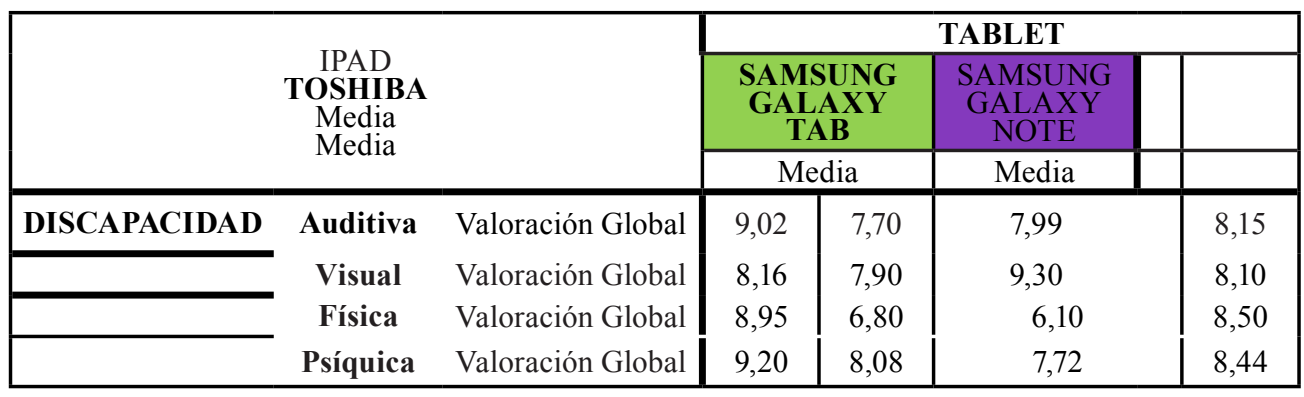

Tabla 4. Valoración global por tipo de discapacidad

Como hemos podido observar en los datos obtenidos, la valoración que los alumnos con discapacidad han hecho de cada tipo de tablets nos ha servido para detectar qué modelo se adapta mejor a las peculiaridades de cada tipo de discapacidad.

Los resultados de la evaluación muestran que, para aquellos alumnos que presentan discapacidad visual les vendría mejor el Samsung Galaxy Tab posicionándolo el número uno en prioridad, con una puntuación de 9,3 sobre 10. Según estos alumnos, el principal inconveniente que detectan en iPad es que no dispone de una tecla específica de retroceso, mientras que Samsung sí que la incorpora, haciendo de esta forma más fácil su manejo. 
Para alumnos con discapacidad auditiva no parecen existir grandes distinciones entre unas tablets u otras, aunque puntúan como preferido al iPad. Por otro lado, tienen menor preferencia sobre el dispositivo portátil Toshiba.

Recordar que las personas con este tipo de discapacidad presentan una serie de dificultades de orientación y comprensión, debido a que su pensamiento está más vinculado a lo concreto y tienen más dificultades para la reflexión abstracta. A simple vista, la preferencia del iPad frente al Toshiba parece radicar en las diferencias de valoración a favor del sistema operativo OSX del iPad señalado mayoritariamente como de mejor usabilidad por estos alumnos que el sistema operativo Android de Toshiba.

Para finalizar observamos que los alumnos con discapacidad física y necesidades educativas especiales prefieren el iPad al resto de tablets, aunque, concretamente para los que presentan discapacidad física, el Samsung Galaxy Note se sitúa muy cerca de iPad, con un 8,5 frente a un 8,95 para dispositivo de Apple. De especial relevancia la incorporación de un "bolígrafo interactivo" beneficia gratamente a personas con manipulación reducida al mejorar su precisión. Además Samsung Galaxy Note presenta un sistema que desactiva el sistema táctil cuando detecta que estamos escribiendo con el S-Pen, y una opción de reconocimiento de contornos o fórmulas que ayuda cuando se dibujen gráficos u operaciones matemáticas, lo cual facilita el uso del tablet para estos alumnos.

\subsection{Evaluación del entorno virtual de la Universidad de Murcia}

A continuación detallamos los resultados obtenidos en el cuestionario en relación, ya no tanto al manejo de las tablets sino a la opinión que los alumnos con discapacidad hacen de la propia página web de la Universidad de Murcia en relación a los servicios que proporciona.

Gráfico 7. Valoración de actividades universitarias on line

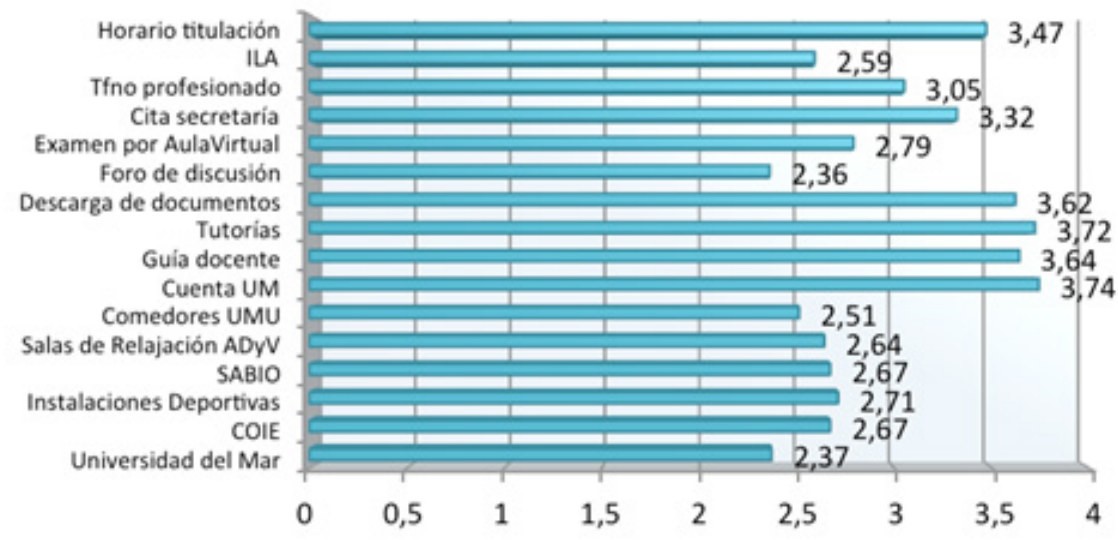


Como podemos observar en el gráfico, dentro de los tres bloques trabajados en el estudio: Servicios Universitarios, Campus Virtual y Facultades, los estudiantes con discapacidad conceden mayor utilidad al Campus Virtual, siendo el acceso a la Cuenta Personal el más votado con una puntuación de 3,74 sobre 4, seguido del "envío de tutorías" con 3,72 y el "acceso a la guía docente de las asignaturas" con una puntuación de 3,64. Por otro lado, el recurso considerado con menor utilidad entre ellos es el "Foro de discusión" con una puntuación de 2,36, seguido del servicio de "Espacios para consumo de comida" con un 2,51.

Por otro lado, las preguntas abiertas nos han proporcionado más información relacionada con la página web de la Universidad y su acceso por parte del alumnado. En concreto se ha detectado una clara necesidad de cambio en la estructura del entorno virtual.

Para la mayoría de los alumnos con discapacidad, hay un exceso de complicación en cuanto al uso de la página, en lo que a temas de estructura y organización de contenidos se refiere. Por ello, la principal demanda que éstos hacen es la de proporcionar un acceso más directo a las cosas, mejora de la claridad de conceptos e iconos de acceso, simplificando los contenidos y haciéndolos más coherentes, como por ejemplo en el acceso a la cuenta personal de la Universidad.

De igual forma, también se demanda una reducción en las opciones de menús, pues actualmente hay muchos menús inactivos o perdidos.

Otras de las sugerencias que hacen los alumnos es que se de mayor facilidad para establecer comunicación con los docentes y los propios compañeros de aula, que haya mayor claridad en cuanto al acceso a la biblioteca y sus diferentes recursos, o incluso que la información relacionada con los "Espacios para consumo de comida propia" debería facilitarse desde todas las facultades, para facilitar el acceso a la información.

\section{Bibliografía}

BERNETE, F. (2010). Usos de las TIC, Relaciones sociales y cambios en la socialización de las y los jóvenes. Revista de Estudios de Juventud, 88. 97-114

BERRÍOS, L.; BUXARRAIS, M. R. (2005): "Las tecnologías de la información y la comunicación (TI C) y los adolescentes. Algunos datos". OEI , Monografías virtuales: Ciudadanía, democracia y valores en sociedades plurales, Número 5 Mayo de 2005.

GUBERN, R. (2000). El eros electrónico. México, Santillana.

LORENTE, S. BERNETE, F.; BECERRIL, D. (2004): Jóvenes, relaciones familiares y tecnologías de la información y de la comunicación. Madrid: Injuve.

OCHAITA, E; ESPINOSA, M. A; GUTIÉRREZ H. (2011). Las necesidades adolescentes y las nuevas tecnologías de la información y la comunicación. Revista de Estudios de Juventud, 92. 87-110 
PÉREZ-DÍAZ, V.; RODRÍGUEZ, J. C. (2008): La adolescencia, sus vulnerabilidades y las nuevas tecnologías de la información y la comunicación. Fundación Vodafone España.

PÉREZ, A. (2011) Escuela 2.0. Educación para el mundo digital. Revista de Estudios de Juventud, 92.63-86.

SOTELO J. (2011). Si McLuhan levantara la cabeza...La computación en la nube y el nuevo paradigma socio cultural. Revista de Estudios de Juventud, 92. 111-122

ZWEIG, J. M.; BUBERSTEIN, L.; MCGINLEY, K. A. (2001). Adolescent health risk profile: the co-ocurrence of health risk among females and males. Journal of Young and Adolescence, 30(6), 707-728.

\section{Los autores}

Ana Millán Jiménez, es Profesora Titular de Universidad en el Departamento de Sociología y Trabajo Social de la Universidad de Murcia. Ha sido investigadora en las Universidades de Glasgow, Chicago y California (Berkeley). Directora del Departamento de Sociología y Política Social desde 2005 hasta 2008. Actualmente es Coordinadora del Servicio de Atención a la Diversidad y Voluntariado de la Universiad de Murcia. Sus líneas de investigación son Sociología de la Comunicación y la Opinión Pública y Sociología de la Diversidad. Entre las últimas publicaciones destacan Las elecciones al parlamento europeo en la Región de Murcia (2011) y Jóvenes, vivienda y beneficios fiscales. Un discurso inacabado (2012). En los últimos años ha coeditado el libro Hacienda pública territorial. Un enfoque Multidisciplinar (2012).

Antonio Pérez Manzano. Dpto. Psicología Evolutiva y de la Educación. Ha participado en numerosas actuaciones y proyectos de investigación que en temáticas tales como actitudes hacia la ciencia y la tecnología, vocaciones científico-tecnológicas, estimulación del pensamiento científico-creativo y televisión educativa. Ha elaborado o colaborado en el diseño de materiales educativos y/o didácticos (la mayoría basados en TIC) para el Ministerio de Educación, el Ministerio de Ciencia y Tecnología, la Real Academia de Ciencias Físicas y Naturales, Compaq España, Sony Entertainment, Santillana, Hasbro Inc., Fundación Española para la Ciencia y la Tecnología (FECYT), Fundación Séneca, Lego Educational, etc. 